\title{
El culto a los Lares Viales en Asturias
}

\author{
Narciso SANTOS YANGUAS \\ Universidad de Oviedo \\ nsantos@uniovi.es
}

\section{RESUMEN}

La romanización implica, desde el punto de vista religioso, una interpretación romana de las divinidades indígenas a través de un proceso de sincretismo. El ejemplo más claro lo hallamos en los dioses indígenas de los caminos, cuya existencia ha llegado hasta nosotros en numerosos epígrafes, donde se les asimila con los Lares Viales romanos. Su número aumenta en suelo asturiano día a día (Tineo, Allande, Lugo de Llanera....), a pesar de que tales monumentos no presentan los foculi (hoyos) para depositar las ofrendas.

Palabras clave: dioses indígenas de los caminos, Lares Viales, proceso de sincretismo, interpretación romana.

\section{The Cult of Lares Viales in Asturias}

\begin{abstract}
Romanization implies, from a religious point of view, a Roman interpretation of indigenous deities by means of a syncretic process. The clearest example is to be found in the indigenous gods of ways, whose existence is known to us through numerous epigraphs and who are assimilated to Roman Lares Viales. Their numbers increase on Asturian soil daily (Tineo, Allande, Lugo de Llanera...) despite the fact that the monuments do not count with «foculi» (holes) for offer deposits.
\end{abstract}

Keywords: indigenous gods of ways, Lares Viales, syncretic process, Roman interpretation.

SUMARIO: 1. Introducción: los Lares en la España romana. 2. Los Lares Viales. 3. Conclusiones.

FECHA DE RECEPCIÓN: 01 DE 11 DE 2011

FECHA DE ACEPTACIÓN: 12 DE 12 DE 2011 


\section{INTRODUCCIÓN: LOS LARES EN LA ESPAÑA ROMANA}

Partimos del hecho de que los Lares fueron los dioses romanos que, junto con Júpiter, tuvieron una mayor aceptación en las provincias hispanorromanas y, de manera especial, en el Noroeste peninsular, en contraste con lo que sucedería en el resto del Imperio ${ }^{1}$.

Cualquiera que haya sido su origen, los Lares se encuentran documentados, casi desde los primeros tiempos de la historia de Roma, como divinidades protectoras de los campos y, sobre todo, de los aspectos domésticos y familiares ${ }^{2}$.

El carácter campestre que los Lares habían adquirido en su origen se manifiesta con claridad en la existencia muy antigua de los cultos realizados en honor de los Lares Compitales ${ }^{3}$.

A este respecto el proceso se habría producido de la siguiente forma: en el momento en que en los compita (encrucijadas) rurales se edificaron sacella (recintos de culto) consagrados en honor de los Lares Compitales el número de estos dioses aumentaría de manera considerable.

Sin embargo, de la misma manera que, en cada una de las casas romanas, no se rendiría culto más que a un solo dios Lar, puesto que en el caso de los compita rurales la realidad correspondería a dos caminos cortándose en ángulo recto, los Lares serían dos ${ }^{4}$.

En cualquier caso la evolución del culto a los Lares en Roma atravesaría al parecer por dos fases históricas distintas:

- una primera, que finalizaría con la República, en la que los Lares conservarían, incluso en las manifestaciones de la religión oficial, el carácter rústico y familiar que responde a la realidad de sus orígenes;

- y una segunda etapa, que se inicia con todo lo que conlleva la reforma religiosa ideada y llevada a la práctica por el primer emperador romano.

Como consecuencia de esta iniciativa reformadora los antiguos dioses de los caminos (lares) pasarían a convertirse en Lares Augusti, lo que implicaba a un mismo tiempo que las ceremonias y ritual de su culto se vinculasen directamente con el emperador y los miembros de la familia imperial, aunque sin pasar a ser por ello el exponente genuino de lo que suponía el culto al gobernante ${ }^{5}$.

\footnotetext{
${ }^{1}$ Al parecer sus cultos no resultarían excluyentes sino, más bien, complementarios, como podemos observar en el caso de la Asturias antigua. cf. N.Santos, «El culto a Júpiter en la Asturias romana: sincretismo y arraigo», L.Hernández (ed.), El mundo religioso hispano bajo el Imperio Romano: pervivencias y cambios, Valladolid, 2007, pp. 249ss.

2 J.Mangas, «Religiones romanas y orientales», Historia de España antigua, tomo II. Hispania romana, Madrid, 1978, pp. 628-629.

${ }^{3}$ L.Adams Holland, «The Shrine of the Lares Compitales», TAPA 68 (1937) 428ss.

${ }^{4}$ En este sentido parece lógico suponer que la pluralidad del concepto de los Lares derivaría de la veneración a estos dioses en las encrucijadas, antes de ser confundidos con los penates.

${ }^{5}$ El culto al emperador entrañaba otras connotaciones, a pesar de lo cual no arraigaría en territorio asturiano con la misma fuerza que el rendido a los dioses de los caminos. Cf. J.Mangas, «El culto imperial en el Noroeste de Hispania», L'Erma di Bretschneider (ed.), Culto imperial: política y poder, Roma, 2007, pp. 705 ss.
} 
Pese a ello, es posible afirmar que la creencia en los Lares como espíritus bienhechores y protectores, de los miembros de la familia especialmente, se hallaría tan arraigada en la conciencia religiosa de los ciudadanos romanos que en algunas regiones del Imperio perduraría incluso tras la desaparición del sistema religioso del que había formado parte originariamente.

El exponente más claro de la pervivencia de este culto hasta el final del Imperio romano parece desprenderse de los ataques que los escritos de los autores cristianos dirigen contra él ${ }^{6}$.

Sin duda la potencia y vigor alcanzados por este culto propio del ámbito doméstico romano derivarían de su temprana adecuación a las características del espíritu religioso romano antiguo.

Esto supondría que, con el transcurrir de los años, tendría lugar todo un conjunto de alteraciones, cambios y añadidos a las ceremonias y cultos que definían la veneración a los Lares, aunque tal vez no se trataría más que de ciertos aspectos externos de una importancia no especialmente significativa, al tiempo que permanecería inalterable la base y el fondo de lo que dicho culto representaba ${ }^{7}$.

De cualquier forma los Lares alcanzarían, en el marco de la religiosidad romana, una amplitud de atribuciones enorme de acuerdo con lo que nos han dejado reflejado en sus escritos los autores antiguos.

Este hecho contribuiría a facilitar su sincretismo y asimilación con las divinidades indígenas en las distintas regiones en las que los romanos asentaron su cultura y organización ${ }^{8}$.

Ahora bien, en el culto rendido a los Lares en territorio peninsular ibérico hemos de hacer una distinción muy clara entre el correspondiente a los Lares Viales y los propios de los Lares romanos y augustos.

En el primero de los casos, como analizaremos más abajo, resulta evidente que, bajo sus nombres, se encubre una realidad religiosa indígena anterior, posiblemente sincretizada, en cuanto al significado de su culto, con los Lares como divinidades típicamente romanas?.

Sin embargo, a nuestro nivel de conocimiento actual no es posible distinguir si las denominaciones indígenas que acompañan a los Lares tópicos y gentilicios es-

\footnotetext{
${ }^{6}$ Tanto más cuanto que no se trata de noticias recogidas de la documentación literaria anterior sino de hechos vividos directamente, que se estaban produciendo en los años de su redacción. Es el caso, por ejemplo, de san Martín Dumiense (De correctione rusticorum 16.4-5): cf. J.E.López Pereira, Cultura, superstición e etnografia de Galicia a través de Martinho de Braga. De correctione rusticorum, A Coruña, 1996.

${ }^{7}$ El momento de su final parece coincidir con el año 392 d.C., cuando, a través de un edicto de Teodosio (Codex Theodosianus XVI.10), se prohibían las ceremonias correspondientes tanto a los lares como a los penates.

${ }^{8}$ Para el caso del Noroeste peninsular remitimos a S.García Martínez, «El panteón indígeno-latino en las tres capitales conventuales del Noroeste hispanorromano: su estudio comparativo», XI Congreso Internacional de Egiprafia Griega y Latina, Roma, 1999, pp. 171ss.

${ }^{9}$ Esta «interpretación romana» de la realidad religiosa indígena parece haber sido común a otras divinidades de origen prerromano. Cf. J.Webster, «Interpretatio: Roman Word, Power and Celtic Gods», Britannia 26 (1995) $153 \mathrm{ss}$.
} 
tán conectadas con una comunidad, un lugar geográfico concreto o una agrupación familiar ${ }^{10}$.

Conocemos en nuestros días que el área de localización de los Lares Viales se identifica con las regiones hispanas en las que la romanización se produciría más tarde, a pesar de que este proceso de asimilación de las formas de vida romana fuera más intenso de lo que se venía creyendo tradicionalmente.

Por lo tanto, y a pesar de la pervivencia de las formas de vida indígenas en dichos territorios y de que dichos Lares encubren sin duda la presencia evidente de antiguas divinidades de la zona, las comunidades septentrionales identificarían al dios romano con el local, debido precisamente a que tenían conocimiento ya de los dioses romanos y entendían las similitudes que guardaban con los suyos propios ${ }^{11}$.

\section{LOS LARES VIALES}

El culto a estas divinidades en territorio peninsular ibérico ha sido objeto de abundantes trabajos por parte de los investigadores contemporáneos, destacando además el hecho de que el número de documentos (epigráficos) vinculados con ellas resulta sorprendente si lo comparamos con lo que sucede en el resto de las provincias del Imperio ${ }^{12}$.

Por otro lado, aunque el contenido religioso del campo epigráfico de las dedicatorias a los Lares Viales trascienda su función de dioses conectados con la protección de las vías de comunicación, su presencia se nos muestra siempre en contextos de estos ejes camineros ${ }^{13}$.

No debemos olvidar, además, que estos dioses asumirían, junto con el culto a Júpiter y lo que ello significaba, un papel especial con respecto al proceso de integración de las comunidades septentrionales en las formas de vida romana ${ }^{14}$.

Junto a ello resulta destacable igualmente el hecho de que unas divinidades tan populares en la religión romana como los Lares de los caminos cuente con un número de testimonios tan exiguo en el resto del Imperio (apenas media docena), lo que hace que el contraste sea todavía más fuerte con respecto al Norte peninsular.

De cualquier forma, las características y paralelismo que presentan las formas de culto a los dioses de los caminos resulta evidente con respecto a otras regiones del arco nordoccidental hispano, en especial en los territorios más próximos a Asturias ${ }^{15}$.

\footnotetext{
${ }^{10}$ Es posible que se refieran exclusivamente a una denominación de lugar (topónimos).

${ }^{11}$ M.I.Portela, «Los dioses Lares en la Hispania romana», Lucentum 3 (1984) 175.

${ }^{12}$ Basten como ejemplo J.C.Bermejo, «Los dioses de los caminos», La sociedad en la Galicia castreña, Santiago de Compostela, 1978, pp. 77ss., y M.I.Portela, «Los dioses Lares en la Hispania romana», pp. 153ss.

${ }^{13}$ A este respecto sobresale la ingente labor, tanto constructora como reparadora, llevada a cabo por los emperadores con respecto a las vías romanas del Noroeste peninsular. Cf., por ejemplo, A.B.Bosworth, «Vespasian and the Provinces. Some Problems of the Early 70's», Athenaeum 51 (1973) 49ss.

${ }^{14}$ En otras regiones de la Península Ibérica más romanizadas esas funciones serían asumidas por el culto imperial.

15 J.Gómez Vila, «Dedicatorias a los Lares Viales en la provincia de Lugo», Gallaecia 23 (2004) 135ss. 
El culto a estas divinidades parece constituir, junto con el rendido a Júpiter, el elemento religioso romano mejor aceptado por parte de las poblaciones septentrionales de la Península Ibérica.

Las manifestaciones religiosas de dicho culto representarían la fase final de la evolución de los lares de las localidades o de las agrupaciones gentilicias correspondientes a las regiones escasamente romanizadas del septentrión hispano ${ }^{16}$.

El hecho de que la existencia de este tipo de divinidades se halle escasamente documentado en el resto de las provincias romanas del Imperio nos lleva a suponer que su concentración en el Norte peninsular ibérico sería debido a que, bajo la advocación de Lares Viales, se hallen encubiertos un conjunto de numina indígenas, que de esta manera perdurarían no solo en tiempos romanos sino incluso en etapas históricas posteriores ${ }^{17}$.

Por su parte otros aseguran que los Lares en general, considerados como dioses latinos, se expandirían desde Roma al Noroeste peninsular en los inicios del siglo II d.C. y servirían como medio de unión y de asimilación con los numina locales (de cada uno de los emplazamientos de habitat) ${ }^{18}$.

De esta manera se daría paso a un proceso de sincretismo entre la religión romana y la religión indígena, que se extiende entre el año 19 a.C. y finales del siglo II de nuestra era, de modo que, cuando ya se había cerrado este proceso, durante el siglo III, es cuando surgen las dedicatorias a los Lares Viales ${ }^{19}$.

Algunos investigadores consideran que esta forma romana de los Lares, además de entrañar una realidad religiosa, encubre en sí misma un carácter indoeuropeo (tal vez céltico), de modo que la presencia romana traería consigo la desaparición de ese espíritu indígena que encubrían ${ }^{20}$.

Eso significa que, a través del culto a los Lares, se produciría el paso desde una sociedad indígena (céltica) a otra céltico-romana o galaico-romana, ya romanizada (o, dicho en otros términos, este culto no supondría más que una fase hacia la romanización completa de los indígenas septentrionales, al tiempo que se transformaría la mentalidad de tales comunidades).

Dicho culto, junto al que hemos de situar igualmente el correspondiente a Júpiter, se halla en la base de la asimilación y del sincretismo entre las religiones locales y la religión romana.

Se explica así el hecho de que divinidades del panteón romano como Mercurio no hayan tenido en Asturias y Galicia más que un papel muy modesto, frente a otras, como Júpiter, los Lares y las ninfas, que se erigirían en los dioses fundamentales a través

\footnotetext{
16 J.M.Blázquez, «Las religiones indígenas del N.O. de la Península Ibérica en relación con Roma», Legio VII Gemina, León, 1970, pp. 65ss.

${ }^{17}$ F.López Cuevillas, «Estudos sobre a Edade do Ferro do noroeste da Peninsula. A relixion», ASEG 6 (1934) 295 ss.

18 S.Lambrino, «Les cultes indigènes en Espagne sous Trajan et Hadrien», A.Piganiol (ed.), Les empereurs romains d'Espagne, París, 1965, pp. 223ss.

${ }^{19}$ D.Plácido, «La conquista del norte de la Península Ibérica: sincretismo religioso y prácticas imperialistas», Marie-Madeleine Mactoux y Evelyne Geny (eds.), Mélanges P.Lévêque, París, 1988, pp. 229ss.

${ }^{20}$ J.Alarçao, R.Étienne y G.Fabre, «Le culte des Lares à Conimbriga», CRAI 1969, pp. 213 ss.
} 
de los cuales el Estado romano buscaría una forma de unificación de la vida religiosa en el cuadrante nordoccidental hispano ${ }^{21}$.

En ese contexto la administración y el ejército se convertirían en los vehículos de transmisión de tales cultos, de acuerdo con las vías de comunicación más representativas y las dedicatorias de carácter oficial.

Ahora bien, la evolución del culto a los Lares en territorio astur y galaico se hallaría al parecer en una fase intermedia, en la que divinidades como Júpiter cumplirían el papel de cohesión e integración que el culto imperial desempeñaría en otras regiones del Imperio ${ }^{22}$.

Y en ese mismo ámbito hemos de situar a los Lares Viales, de manera que, tras haber asumido a ciertos dioses locales, pasarían a convertirse en divinidades de carácter oficial, siendo su figura y funciones comparables a las de los Lares Augusti ${ }^{23}$.

No debemos olvidar a este respecto que el culto a estos Lares, que se hallaba conectado directamente con el que se rendía a los emperadores, arraigaría exclusivamente en las regiones más intensamente romanizadas.

Mientras tanto, en el Noroeste romano en general sería únicamente el culto rendido a los Lares Viales el que contribuiría a ese proceso de cohesión de las poblaciones indígenas con el mundo romano, dado que en dicha zona el culto imperial no se desarrollaría de manera profunda ${ }^{24}$.

En otros términos: el localismo implícito en las invocaciones a estos espíritus protectores de los caminos nos lleva a pensar que, bajo dichas denominaciones, se esconde un numen (o numina) astures y galaicos, sincretizados (asimilados) o suplantados por estos Lares.

Dichos cultos sobrevivirían durante mucho tiempo (incluso durante los primeros siglos del cristianismo) de acuerdo con lo que nos testimonia Martín de Dumio ${ }^{25}$, quien recomienda que no se enciendan hogueras en los cruces de caminos (trivia).

Esta práctica se ha mantenido a lo largo de los siglos, y no solo en los cruceiros y/o amilladoiros del Norte peninsular sino también en las rogativas que en territorio castellano-leonés se han venido realizando hasta hace escasas décadas con el fin de impetrar la lluvia por ejemplo (procesiones con el santo protector a la cabeza).

Por otro lado, en ocasiones se ha asegurado que el culto a los Lares Viales ofrece un cierto paralelismo con el correspondiente a las Matres en territorio galo, en especial cuando unos y otras son invocados con los calificativos de biviae, triviae y quadriviae ${ }^{26}$.

${ }^{21}$ M.I.Portela, «Los dioses Lares en la Hispania romana», p. 166.

22 J.Nichols, «Indigenous Culture and the Process of Romanization in Iberian Galicia», AJPh 108 (1987) 129 ss.

${ }^{23}$ Tal vez esa evolución hubiese conducido a un asentamiento más intenso del culto imperial en dichas regiones; sin embargo, el desfase cronológico no permitiría llevar a cabo dicho proceso al verse enfrentado dicho culto con el cristianismo.

${ }^{24}$ F.Acuña, «Los Lares Viales en la Galicia romana», Actas do II Congreso Nacional de Arqueología, Coimbra, 1971, pp. 351ss.

${ }^{25}$ De correctione rusticorum 16.4-5.

${ }^{26}$ Sin embargo, las Matres parecen relacionarse más bien con la fecundidad, pudiendo ser consideras por ello como una especie de diosas madres. Cf. M.I.Portela, «Los dioses Lares en la Hispania romana», p. 167. 
Más fácil resulta comparar las semejanzas que existen entre los Lares Viales de Galicia y Asturias con otros dioses de tipo indoeuropeo (céltico) como Mercurio, Lug..., aunque el modelo de los Lares Viales es posible que tengamos que buscarlo en el Hermes griego ${ }^{27}$.

\subsection{LARES VIALES Y PROCESO DE SINCRETISMO}

Tal vez el ejemplo más claro de este proceso de sincretismo (adaptación y/o interconexión entre divinidades y cultos indígenas y romanos) lo constituyan los Lares Viales, que se nos presentan en número considerable en territorio galaico ${ }^{28}$, y en menor proporción en suelo astur.

A este respecto hemos de tener en cuenta que, del mismo modo que sucede con relación a otras muchas expresiones de la religiosidad astur-romana, el ámbito geográfico de análisis que nos proponemos llevar a cabo en las páginas siguientes con respecto a los dioses de los caminos debe ampliarse a todo el territorio ocupado por los astures $^{29}$.

Si tenemos presente que los caminos y vías de comunicación en general, debido a su propia naturaleza, se convertían en lugares peligrosos para quienes los frecuentaban, no puede extrañarnos el hecho de que una capilla, un ara, un pequeño santuario o un edificio sagrado de dimensiones no excesivamente amplias configuraran elementos de orientación sumamente apreciados por parte de los viajeros y caminantes.

Por ello, a pesar de que desconozcamos en gran medida las denominaciones indígenas de tales divinidades vinculadas a los viandantes, han llegado hasta nosotros en abundantes epígrafes hallados en todo el cuadrante nordoccidental hispano, asimiladas con los romanos bajo el apelativo de Lares Viales $^{30}$.

En el caso concreto de los astures transmontanos el número de ejemplos de esta naturaleza va en aumento a medida que transcurren los años.

Así destacamos, en primer lugar, la inscripción asignada en un principio a Santianes de Tuña (concejo de Tineo), dedicada por Quincto Publio y que, de acuerdo con Manzanares ${ }^{31}$ habría que localizar realmente al oeste de la actual población de Tuña (CIL II.5734):

\section{$Q \quad P$ \\ LARIBVS \\ VIALIBVS \\ EX VOTO}

\footnotetext{
27 J.C.Bermejo, La sociedad en la Galicia castreña, pp. 176-177.

${ }^{28}$ Ver, entre otros, J.Taboada, «Nuevos testimonios del culto a los Lares Viales en Gallaecia», Gallaecia 2 (1976) 193ss.

${ }^{29}$ Ver, por ejemplo, J.Mangas, «El culto imperial en el Noroeste de Hispania», pp. 710-712.

${ }^{30}$ J.C.Bermejo, La sociedad en la Galicia castreña, pp. 177 ss.

${ }^{31}$ Dos aras, una inédita, a los Lares Viales en Tuña (Tineo-Asturias), Oviedo, 1986, pp. 6-7.
} 
Q(uinctus) $P($ ublius?)/Laribus/Vialibus/ ex voto.

«Quincto Publio (lo dedica) a Los Lares Viales de acuerdo con su promesa».

Dicho epígrafe en la actualidad se halla desaparecido, aunque los datos de que disponemos con respecto a su descubrimiento y existencia (Martínez Marina, Vigil, Somoza) lo relacionan con un puente romano sobre el río Narcea en el marco territorial de la parroquia tinetense de Tuña ${ }^{32}$.

Bajo la denominación genérica de Lares Viales es posible que se encubran en este caso (y en otros) referencias a divinidades de carácter local, sincretizadas con estos dioses romanos de los caminos, con los que se fusionarían al tiempo que harían desaparecer sus advocaciones anteriores ${ }^{33}$.

A este primer documento hemos de añadir otro, descubierto en el año 1983 en una casa de la misma localidad del concejo tinetense, al que se refiere Manzanares, casi de forma monográfica, en el trabajo anteriormente mencionado ${ }^{34}$.

Se trata de una piedra arenisca, cuyas medidas corresponden a 59 X 25 X $13 \mathrm{cms}$., sin ninguna fragmentación en su estructura, de manera que se puede reconstruir perfectamente su campo epigráfico, acoplado a la parte superior de la pieza:

$\begin{array}{ll} & \text { SEM CAS } \\ & \text { LARIBVS } \\ & \text { VIALIBVS } \\ & \text { EXVOTO } \\ & \text { SACRVM }\end{array}$

Sem(pronius) Cas(sius)/Laribus/Vialibus/ ex voto/ sacrum.

«Consagrado a los Lares Viales. Sempronio Cassio (lo dedicó) de acuerdo con su promesa».

En este monumento sobresale el hecho de que se nos testimonia con toda claridad el nombre de la persona que dedica el altar (junto con su inscripción) a estas divinidades de los caminos, tratándose ya, tal vez porque su cronología correspondería a una fase avanzada del siglo II, de un individuo latinizado en cuanto a sus antropónimos (Sempronius Cassius) ${ }^{35}$.

Junto a ello hemos de destacar igualmente la lápida votiva hallada en la localidad de Comba (concejo de Allande), en el límite con el territorio de Cangas del Narcea, dedicada posiblemente por un personaje de nombre Próculo.

\footnotetext{
${ }^{32} \mathrm{Su}$ cronología parece relacionarse ya con el siglo II d.C., lo que tal vez corrobore la misma nomenclatura de los antropónimos del dedicante.

${ }^{33}$ F.Diego Santos, Epigrafía romana de Asturias, Oviedo, 1985, no 5, pp. 36-37.

${ }^{34}$ Dos aras, una inédita, a los Lares Viales en Tuña (Tineo-Asturias), pp. 2-5.

${ }^{35}$ En su parte superior presenta los 3 hoyos (foculi) característicos de este tipo de monumentos votivos, en los que se daba acogida a los elementos de ofrenda a los dioses de los caminos para impetrar su protección en el recorrido de los trazados viarios correspondientes.
} 
Nos encontramos ante un documento epigráfico descubierto en el año 1944 por D.Ramón Losas Fernández en un cortinal de la población mencionada del concejo allandés, siendo adquirida 30 años después por D. Antonio García Linares, quien la estudió y publicó hace más de un cuarto de siglo ${ }^{36}$.

El contenido de su campo epigráfico, aunque el soporte de la inscripción parece hallarse algo fragmentado, se halla claramente expresado ${ }^{37}$ :

\section{LARIBVS \\ VIALIB \\ VS PRO}

\section{Laribus/Vialib/ us Pro(culus)}

«A los Lares Viales (lo dedica) Próculo».

Coincidiendo con el ámbito geográfico en que se descubrió esta estela se hallaron igualmente restos de antiguos aprovechamientos auríferos por parte romana, por lo que es posible que la dedicatoria estuviera realizada por algún componente de la mano de obra utilizada en las labores correspondientes a esta zona del río Comba (explotaciones de Comba, Iboyo, Abaniella, Peruyeda.... ${ }^{38}$.

En cuanto a la reconstrucción del final de la tercera línea, si admitimos que el campo epigráfico esta completo, podemos aceptar perfectamente que, bajo el término $P R O$, estaría presente el dedicante de la inscripción, que se identifica con un tal Pro $(\text { culus })^{39}$.

A este respecto contamos con otra serie de antropónimos conservados en documentos epigráficos del territorio de los astures augustanos o cismontanos, como es el caso de Aurelius Pro(culus) en la inscripción leonesa de Liegos ${ }^{40}$ o el de Attius Pro(culus), registrado en el hallado igualmente en territorio de León (en concreto en la región vadiniense de Cármenes $)^{4}$.

Finalmente, por lo que respecta al territorio de la antigua Asturias, contamos con la estela descubierta en el enclave actual de Lugo de Llanera (antigua población romana de Lucus Asturum) en el transcurso de la campaña de excavaciones del año 1989

\footnotetext{
${ }^{36}$ A.García Linares, «Una lápida romana a los Lares Viales en Comba (Allande)», BIDEA 107 (1982) 773 ss.

37 J.Mangas, «La difusión de la religión romana en Asturias», Ministerio de Cultura-Universidad de Oviedo (ed.), Indigenismo y romanización en el conventus Asturum, Oviedo-Madrid, 1983, p. 172.

${ }^{38}$ N.Santos, «Las explotaciones romanas de oro de la cuenca del Arganza en el concejo de Allande (Asturias)», SHHA 20 (2002) 201ss.

${ }^{39}$ F.Diego Santos, Epigrafia romana de Asturias, nº 5a, pp. 38-39.

${ }^{40}$ CIL II.5718. Cf. M.A.Rabanal y S.García Martínez, Epigrafía romana de la provincia de León: revisión y actualización, León, 2001, nº 383, pp.413-414.

${ }^{41}$ A.E. 1967, n ${ }^{\circ}$ 241. Cf. J.M.Navascués, «La estela funeraria de Cármenes», AEA 43 (1970) 175ss., y M.A.Rabanal y S.García Martínez, Epigrafia romana de la provincia de León: revisión y actualización, $\mathrm{n}^{\circ} 351$, pp. 384-385.

${ }^{42}$ R.Cid, «La lápida a los Lares Viales de Lugo de Llanera (Asturias)», Nuestro Museo 2 (1998) 187ss.
} 
ARAM

LARIBV

VIALIBV

Aram/Laribu(s)/Vialibu(s)

«Ara (consagrada) a los Lares Viales».

Este ara votiva, al igual que sucede con el resto de las dedicadas a los dioses romanos de los caminos, cuenta con un campo epigráfico muy escueto, en el que tal vez resulte extraño, desde el punto de vista gramatical, la utilización del acusativo (aram), que nos lleva a pensar en la presencia de un hipotético dedicante, cuyo nombre desconocemos por no aparecer consignado en la actualidad en el texto de dicha inscripción ${ }^{43}$.

No podemos olvidar a este respecto la importancia lograda por este centro asturromano en relación con las vías de comunicación, tanto en el trazado que lo unía a Gigia (Gijón) como en el que se desplazaba hasta alcanzar la capital de los galaicos lucenses (Lucus Augusti) $)^{44}$.

Sin embargo, ninguna de ellas resulta comparable a la encontrada en la provincia de Lugo (en la actualidad en el Museo del castro de Viladonga), cuyo campo epigráfico se ciñe escuetamente a la fórmula general de la dedicatoria a los Lares Viales (Laribus Vialibus ex voto), sin hacer mención alguna del dedicante (o dedicantes) de dicha estela ${ }^{45}$.

Este monumento destaca por su perfecta conservación y por ofrecernos en su parte superior las cavidades características (foculi) que pudieron ser utilizadas como depósitos de ofrendas, o bien conectarse con la tríada vinculada a la mención de los Lares sin más.

De cualquier forma este tipo de creencias en dioses protectores de encrucijadas y caminos, y en especial de quienes transitaban por ellos, parecen haber permanecido vigentes a lo largo de todo el Imperio romano, puesto que san Martín de Dumio nos asegura, ya en tiempos bajoimperiales muy avanzados (y en un contexto estrictamente cristiano), que se hacía preciso condenar estos cultos conectados con los dioses de las encrucijadas como prácticas netamente paganas (De correctione rusticorum 16.4-5: «Pues encender velas junto a las rocas, los árboles, las fuentes y las encrucijadas de los caminos, ¿qué otra cosa puede ser sino cultura del diablo?»).

\section{CONCLUSIONES}

Ya hemos hecho mención en las páginas precedentes a la difusión alcanzada por los Lares Viales y su conexión con las vías romanas de comunicación más importantes en el ámbito territorial de la Asturias romana.

${ }^{43}$ R.Cid y otras, «Lucus Asturum y un ara inédita a los Lares Viales en Lugo de Llanera (Asturias)», Gallaecia 13 (1994) 113ss.

${ }^{44}$ N.Santos, «Lucus Asturum y las vías romanas de comunicación», Asturias, los astures y la administración romana durante el Alto Imperio, Oviedo, 2009, pp. 521-526.

${ }^{45}$ No se encuentra recogida en el recopilatorio de J.Gómez Vila [«Dedicatorias a los Lares Viales en la provincia de Lugo», 135ss.] entre las correspondientes a la provincia de Lugo. 
De la misma manera hemos destacado el proceso de sincretismo a que se daría paso entre estas divinidades romanas de los caminos y los dioses indígenas a los que de forma tradicional se les había venido encomendando esas mismas funciones de protección de los viandantes.

Por otro lado no podemos negar taxativamente la naturaleza prerromana (indoeuropea o céltica) de las divinidades que se asimilarían con los Lares Viales, aunque tampoco disponemos de indicios suficientes para poder afirmarlo con tanta certeza como han hecho algunos investigadores de nuestros días.

Y menos aún si tenemos en cuenta que el celtismo a ultranza de las comunidades indígenas del septentrión hispano (en especial las galaicas) continúa poniéndose en duda en la actualidad ${ }^{46}$.

De este modo, aun cuando es posible admitir la presencia de comunidades de origen celta (indoeuropeo) en el Noroeste peninsular, su influencia sobre el conjunto de la población y la cultura prerromanas no parece haber sido tan intensa y sustitutiva, dado que lo genuino de las mismas se relaciona con la existencia de un sustrato étnico-cultural prehistórico, que se configuraría y consolidaría durante la fase del Bronce atlántico ${ }^{47}$.

Pero es que, además, este hecho contrasta todavía más si lo comparamos con otras regiones consideradas célticas puras, como son los casos de Galia y/o Britania, que no presentan testimonio alguno del culto rendido a los Lares Viales.

Sin embargo, contamos con un hecho perfectamente constatado: la tipología que descubrimos en buena parte de las dedicatorias votivas a estos dioses de los caminos ofrecen características típicamente romanas, como por ejemplo los foculi (generalmente 3) que aparecen en la parte superior de las aras.

En resumen, es posible afirmar que, bajo un fondo indígena en el que tendría acogida un culto a las divinidades de los caminos y encrucijadas ${ }^{48}$, se superpondría la influencia romana, dando paso así a ese sincretismo que aflora en los Lares Viales, cuyo culto adquiriría un significado intensamente romanizado y romanizador a un mismo tiempo.

Y estas prácticas religiosas no confrontarían con las correspondientes a Júpiter, pues en relación con esas mismas vías de comunicación antigua disponemos en Asturias de varios monumentos dedicados al dios principal del panteón romano.

Frente a ello podría resultar extraña la casi nula existencia de referencias al culto imperial, por lo que no podemos considerar que este elemento amalgamador del espíritu cívico romano arraigase con fuerza en detrimento del correspondiente a Júpiter.

Por lo que se refiere a los dedicantes de este tipo de aras para impetrar la protección de los dioses de los caminos, aunque sin duda el interés sería generalizado, es decir compartido por todos los miembros de las comunidades que se comunicaban entre sí a través de estos trazados viarios en cuyas encrucijadas se erigían dichos altares,

\footnotetext{
46 Ver, por ejemplo, F.Pereira, «O mito celta na historia», Gallaecia 19 (2000) 311ss.

${ }^{47}$ N.Santos, Asturias, los astures y la cultura castreña, Oviedo, 2006, p. 121.

${ }^{48} \mathrm{Sin}$ tener que pensar que se trataría necesariamente de una religiosidad céltica.
} 
no parecen coincidir con colectividades o grupos en su conjunto sino más bien con individuos o personas particulares (Quinto Publio, Sempronio Cassio, Próculo...) ${ }^{49}$.

Finalmente hemos de destacar el hecho de que los altares consagrados a los Lares Viales hallados en Asturias durante la Antigüedad se conectan en su totalidad con vías de comunicación de cierto relieve, que en su mayor parte encubrirían un sentido económico.

Algunas de ellas estarían vinculadas a los distritos de explotación de los recursos mineros (las dos de Tuña-Tineo- y la correspondiente a Comba -Allande-) mientras que otras se conectarían con los aprovechamientos de las condiciones agrícolas (o agropecuarias) de la región (por ejemplo la de Lugo de Llanera en dirección al centro administrativo astur-romano de Gigia-Gijón-) ${ }^{50}$.

Esto significa que, puesto que hasta la actualidad no se han descubierto restos de miliarios en territorio de Asturias, si hacemos excepción de uno hipotético dedicado a Numeriano (que tendría además como referente el enclave de Lucus Asturum), estos documentos dedicados a las divinidades de los caminos nos ayudan a comprender el trazado y significado de los principales caminos de comunicación durante la época romana.

Por último hemos de tener en cuenta una realidad: ninguna de la media docena de aras que acabamos de describir (con sus respectivas dedicatorias) hace referencia a posibles espacios sagrados o edificios religiosos vinculados con ellas, que, en el caso de los Lares Viales, no debieron ser muy amplios.

Tampoco contamos con representación escultórica alguna de dichas divinidades, lo que no resulta muy extraño dado el carácter implícito en el significado de las mismas como orientadoras, vigilantes y protectoras de las vías romanas de comunicación.

Si añadimos a ello el hecho de que desconocemos igualmente si los monumentos mencionados constituirían un fiel reflejo de las ofrendas, sacrificios y libaciones a estos dioses de los caminos, cuyos depósitos podían realizarse en los foculi de la parte superior de las aras, ¿nos encontramos ante una ausencia casi absoluta de lugares de culto consagrados a los mismos en todo el cuadrante nordoccidental hispano?

Aunque la respuesta no implique un no tajante con respecto a otros cultos de tiempos romanos en el caso de Asturias (como Júpiter por ejemplo), todo apunta a que los Lares Viales no contarían con santuarios o templos de entidad significativa, pudiéndose pensar más bien en la existencia de capillas y/o nichos de reducidas dimensiones, ubicados en los cruces de los caminos y en los que se celebrarían los cultos y ceremonias correspondientes.

\footnotetext{
49 A este respecto no debemos olvidar que la seguridad y protección de los viandantes se hallarían en la base de los cultos relacionados con estas divinidades.

${ }^{50}$ N.Santos, «Avance al estudio de la ciudad romana de Lucus Asturum (Lugo de Llanera, Asturias)», J.Mangas y M.A.Novillo (eds.), El territorio de las ciudades romanas, Madrid, 2008, pp. 425ss.
} 